\title{
ANTIBACTERIAL ACTIVITY OF VITEX NEGUNDO LEAVE EXTRACT AGAINST METHICILLIN RESISTANT STAPHYLOCOCCUS AUREUS (MRSA)
}

\author{
A. G Triveni, Suresh kumar Mendem, Channappa T. Shivannavar and Subhaschandra M. Gaddad* \\ Department of P. G. Studies and Research in Microbiology, Gulbarga University, Kalaburagi-585106, \\ Karnataka, India,
}

\begin{abstract}
*Corresponding Author Email: smgaddad@gmail.com
ABSTRACT

Objective: MRSA has threatened the hospital and community settings because of their severity of infections and fast acquiring MDR status. Ethnomedicine as a therapeutic approach has promising solutions in the treatment of skin infections caused by Staphylococcus aureus. In the present study, we have evaluated the antibacterial activity of Vitex negundo against Methicillin Resistant S. aureus (MRSA). Methodology: Extracts from the leaves of V. negundo were prepared by maceration method using polar and non-polar solvents. MRSA ATCC 43300 culture was obtained from Himedia (India). Antibacterial activity was determined by Agar well diffusion method. Results: Both polar and non-polar solvent extracts of $V$. negundo showed significant antimicrobial activities against MRSA. Highest activity of $19 \mathrm{~mm}$ was observed for $n$-hexane extract which is higher than the zone observed for vancomycin. Similarly, ethyl acetate, pet ether and acetone extract showed very good activity, while methanol and chloroform extracts were moderately active. This is the first report on antibacterial activity of Vitex negundo extracts against MRSA. Conclusion: Ethnomedicine is an alternate approach in exploring bio-control agents. Investigation of medicinal plant compounds in finding the newer targets to overcome the pathogenicity of disease causing S. aureus could be the base for future research.
\end{abstract}

\section{KEY WORDS}

MRSA, V.negundo, Antibiotic activity, S.aureus, n-Hexane

\section{INTRODUCTION}

S. aureus is an opportunistic pathogen causes a variety of infections ranging from simple boils to notorious osteomyelitis and endocarditis and is a major cause of hospital and community acquired infections [1, 2]. During the 1950's, Methicillin was one of the efficient antibiotic used in the treatment of invasive $S$. aureus [3]. Within one-decade MRSA has evolved and first reported in the US in 1961. Invasive $S$. aureus has worsened the hospital settings causing severe MRSA infections. Eradication of MRSA has become difficult, due to its extraordinary ability of acquiring resistance against antibiotics used in the treatment of infections (4).

$V$. negundo belongs to Verbenaceae family and is a medicinally important plant [5]. Verbenaceae plant species have many benefits in healing a wide spectrum of health and skin disorders. Herbal formulations of Vitex are available in the market. $V$. negundo exhibits anti-inflammatory, antibacterial and antifungal properties [6]. Hence it is used as an effective bio-control agent in the treatment of skin infections.

Glycopeptides classes of antibiotics are the only advanced treatment options for MRSA infections [7]. The cost of the treatment is not affordable for the low income countries like India. Before the dawn of modern drugs, traditional Ayurvedic therapies were the only source of cost effective treatment for various diseases. The World Health Organization estimates that $80 \%$ of the people in the developing countries rely on traditional medicines [8]. Many tropical treatments used for variety infections are of plant origin. Plant derived drugs have significantly contributed to the development of anti-infectious agents and takes the lead in clinical trials [9]. A report shows $70 \%$ of Indian population relies on plant based 
drugs [10]. Many medicinal plants are unexplored and still remained as a mystery.

Reports on antibacterial activity of $V$. negundo against multi drug resistant pathogens have been poorly studied in India. This is the first report on the antibacterial effect of $V$. negundo against Methicillin Resistant S. aureus (MRSA).

\section{MATERIALS AND METHODS:}

Fresh, healthy leaves of $V$. negundo growing locally (Fig:-1a) were collected. The plant was identified with the help from the data available in the Department of Botany, Gulbarga University, Kalaburagi.

\section{Extraction:}

Plant Leaves were washed under running tap water followed by washing with distilled water and air dried under shade at room temperature. Further pulverized to a fine powder and stored in air tight polyethylene bags. Six different solvents namely Ethanol, Methanol, Acetone, n-Hexane, Petroleum ether and Chloroform were used for preparing the plant extracts.

Fig.1. The plant V.negundo (a) and collection of the plant extracts (b, c)

a)

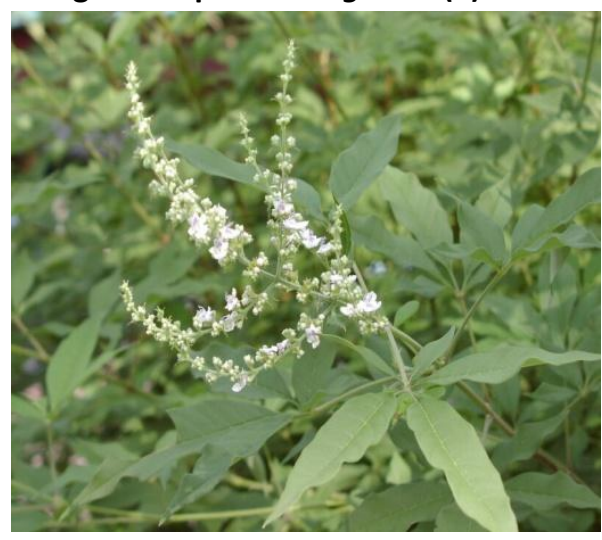

b)

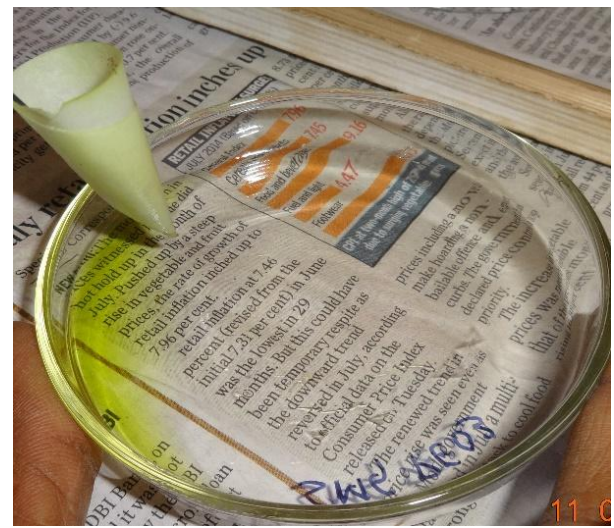

c)

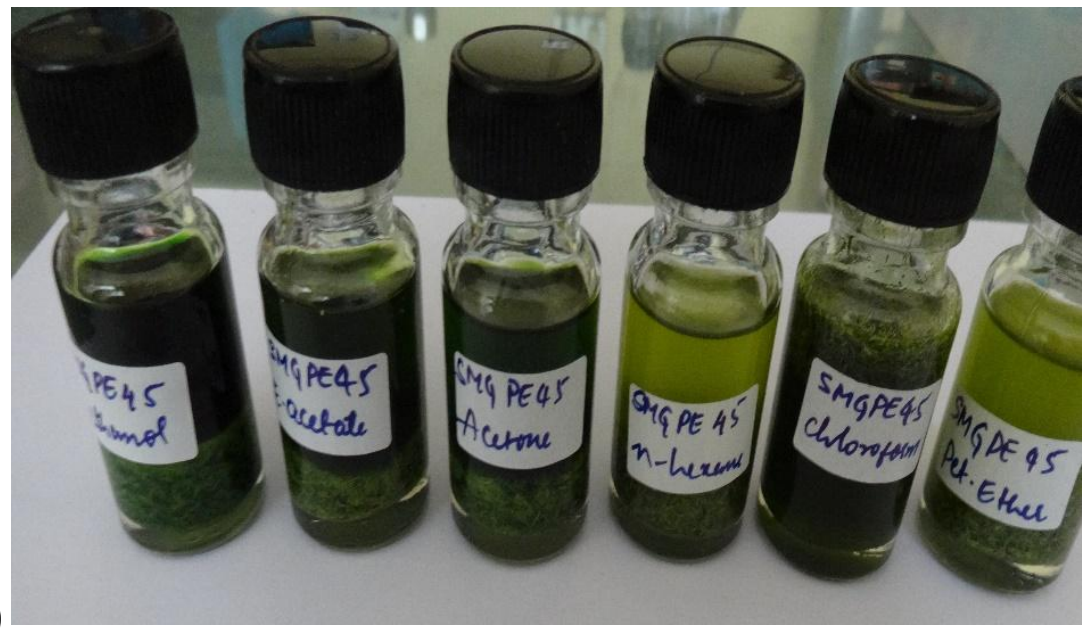

One gram of dried plant material was dissolved in $10 \mathrm{ml}$ of solvent in air tight bottles (Fig 1c). The mixture was maintained on an orbital shaker at $120 \mathrm{rpm}$ in dark for 3 days. The solution obtained was filtered and the solvent was evaporated overnight (Fig 1b). The extract obtained was dissolved in $500 \mu \mathrm{l}$ of DMSO and transferred to a sterile screw cap glass vial.

\section{Antimicrobial susceptibility testing:}

Following antimicrobial discs (Hi media, India) were used in the study: Penicillin (10units), Oxacillin (1mcg), Cefoxitin (30 mcg), Amoxyclav (Amoxycillin / Calvunicacid) (AMC) (20mcg), Cefotaxime (30 mcg), Ceftazidime (30 mcg), Cefpodoxime $(10 \mathrm{mcg})$, Amikacin (AK) 30mcg, Vancomycin (30 mcg), Ciprofloxacin , Doxycycline (DO), Tetracycline (TE), and Tobramycin 
(TOB), Antimicrobial susceptibility testing was performed as per CLSI (2014) guidelines (11).

\section{Microorganisms:}

Staphylococcus aureus (ATCC 43300) was obtained from Hi-media, India. A few MRSA and MDR isolates available in our lab were also used.

Screening of antibacterial activity of the Plant extracts: Antibacterial test was performed by agar well diffusion method [12]. Bacterial suspension was enriched in brain heart infusion broth for overnight. Cultures of 0.5 McFarland were aseptically swabbed over sterile Mueller Hinton Agar plates. Using $5 \mathrm{~mm}$ sterile cork borer, the agar gel was punctured; $100 \mu \mathrm{l}$ of plant extract was pipetted into the wells. Only DMSO served as control. Inoculated plates were incubated at $37^{\circ} \mathrm{C}$ for overnight. The zone of inhibition formed around the wells was measured. Plates were prepared in triplets and the mean diameter of the zone of inhibition was noted.

\begin{abstract}
RESULTS:
The antibacterial activity of V.negundo extracts with different solvents against the MRSA isolates is shown in Table 1: .The non polar solvent, $n$-hexane has showed highest zone of inhibition of $19 \mathrm{~mm}$ against the local MRSA isolate and $20 \mathrm{~mm}$ against the standard ATCC MRSA culture. Petroleum-ether extract showed $17 \mathrm{~mm}$ zone of inhibition against both the MRSA strains. These two observations are greater than the inhibition zones observed with respect to vancomycin (16 $\mathrm{mm}$ ). Similarly, ethyl acetate and acetone extracts showed 16 $\mathrm{mm}$ zone of inhibition, while the activity of methano and chloroform extracts was lower with 12 and $11 \mathrm{~mm}$ zone of inhibition respectively (Fig: 2 ).
\end{abstract}

Table 1: Anti MRSA activity of the solvent extracts of V.negundo

\begin{tabular}{lll}
\hline SI.No & Solvents / antibiotics & S. aureus (MRSA) \\
\hline 1 & Ethyl acetate(100 $\mathrm{L}$ ) & $16 \mathrm{~mm}$ \\
2 & Methanol & $12 \mathrm{~mm}$ \\
3 & Acetone & $16 \mathrm{~mm}$ \\
4 & Petroleum-ether & $17 \mathrm{~mm}$ \\
5 & N-Hexane & $19 \mathrm{~mm}$ \\
6 & Chloroform & $11 \mathrm{~mm}$ \\
7 & Penicillin.G(P) & 13 \\
8 & Oxacillin(ox) & no zone \\
9 & Cefoxitin(cx)/cephoxitin & no zone \\
10 & Amoxyclav(Amoxycillin/Calvunic acid)(AMC) & 15 \\
11 & Cefotaxime(CTX) & no zone \\
12 & Ceftazidime(CAZ) & no zone \\
13 & cefpodoxime(CPD) & no zone \\
14 & Vancomycin & no zone \\
15 & Amikacin(AK) & no zone \\
16 & Tobramycin(TOB) & no zone \\
17 & Tetracycline(TE) & no zone \\
18 & Doxycycline(DO) & 11 \\
19 & Ciprofloxacin(CIP) & no zone \\
\hline
\end{tabular}

Fig.2. The Plates showing the anit MRSA activities of the various antibiotics and the different solvent extracts of the plant V.negundo 


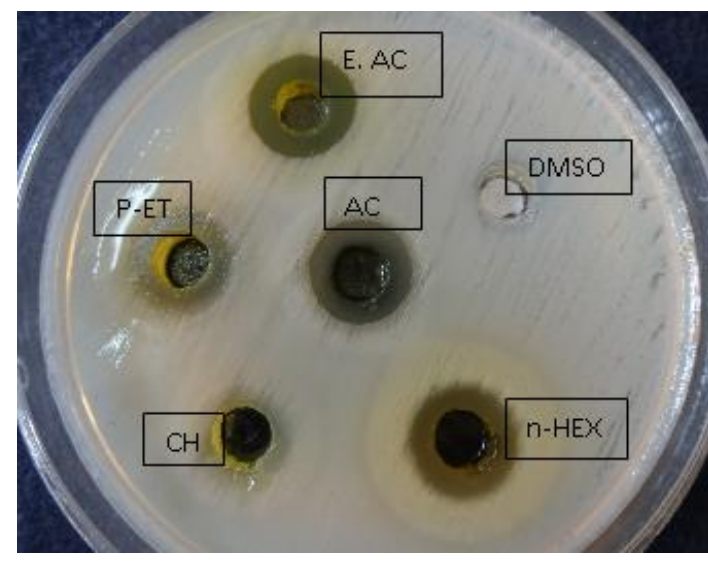

\section{DISCUSSION:}

Extensive and indiscriminate use of antibiotics used for the treatment of infectious diseases is leading to the widespread emergence of drug resistance among etiological agents and an alarming situation of increasing MDR incidences. Treatment to this type of etiological agents and associated infections is influenced by various other factors such as high cost and severe side effects. Herbal medicine has a long history of healing effects and more over a promising alternative medicine. Most of Asian countries are still using phyto-medicine (13). Vitex is widely spread all over India in forest areas, wasteland areas. And various parts of Vitex are being used for different formulations in herbal medicine preparations (14).

$S$. aureus is an opportunistic pathogen, causing an array of infectious diseases and is a leading cause of concern both in hospital and communal healthcare settings. Treatment options have increasingly becoming limited with the rise of wide spread drug resistant strains. In India various reports indicate that MRSA accounted for $30-80 \%$ of infections (15). Vancomycin was the drug of choice used to treat infections associated with multidrug resistant MRSA strains. However, recent decade has witnessed the emergence of VRSA strains which poses serious threat to public health care settings worldwide (16)

Plant materials have been used as a good source of natural therapeutic remedies and are used to treat various infectious diseases in many developing countries. In the present study we have observed a significant anti MRSA activity by the various solvent extracts of the local plant $V$. nrgundo. More

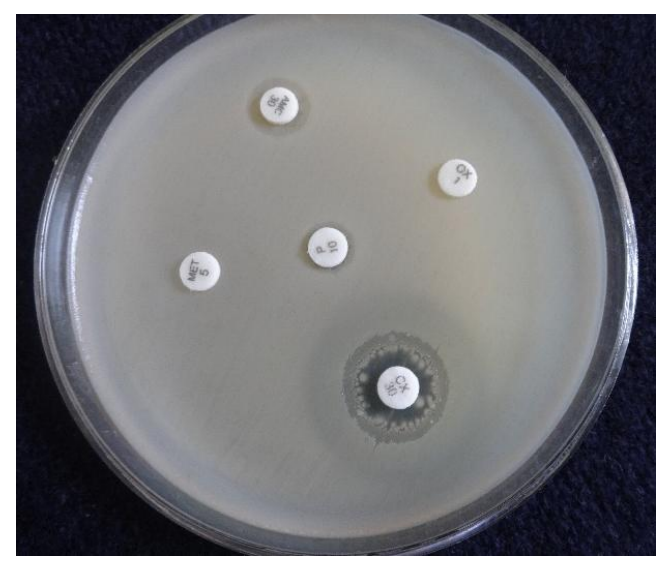

specifically the $\mathrm{n}$-hexane and the pet-ether extracts have exhibited highly promising antibacterial activity, even greater than that observed for some of the antibiotics like vancomycin [17]. Studies on phytochemical analysis of $V$. negundo indicates the presence of high amounts of flavanoids and other bioactive molecules such as saponins, tannis, steroids and reducing sugars (18). Kamruzzaman et al., (2013) have reported wide range activity of V.negundo extracts on various types of etiological agents (19). It appears that ours is the first report on the anti MRSA activities of the plant V.negundo.

\section{CONCLUSION}

Our study reveals significant activities of the $V$. negundo extracts against MRSA and calls for further exploration of phytomedcine from this plant as natural alternative remedy.

\section{REFERENCES}

1. Tong SY, Davis JS, Eichenberger E, Holland TL, Fowler VG. Staphylococcus aureus infections: epidemiology, pathophysiology, clinical manifestations, and management. Clin. Microbiol. Rev, 28(3):603-61,(2015).

2. Klein E, Smith DL, Laxminarayan R. Hospitalizations and deaths caused by methicillin-resistant Staphylococcus aureus, United States, 1999-2005. Emerg Infect Diseases, 13(12):1840, (2007).

3. Enright MC, Robinson DA, Randle G, Feil EJ, Grundmann $H$, Spratt BG. The evolutionary history of methicillinresistant Staphylococcus aureus (MRSA). Proc. Natl. Acad. Sci. U.S.A. 99(11):7687-92, (2002). 
4. Ventola, C. L. The Antibiotic Resistance Crisis: Part 1: Causes and Threats. Pharmacy and Therapeutics, 40(4), 277-283, (2015).

5. Vishwanathan AS, Basavaraju R. A review on Vitex negundo L-a medicinally important plant. Eur J Biol Sci., 3(1):30-42,( 2010).

6. Anita Rani and Anupam Sharma .The genus Vitex: A review. Pharmacogn Rev. 7(14): 188-198, (2013).

7. Shearer MC, Giovenella AJ, Grappel SF, Hedde RD, Mehta RJ, Oh YK, Pan CH, Pitkin DH, Nisbet LJ. Kibdelins, novel glycopeptide antibiotics . I Discovery, production, and biological evaluation . J. Antibiot. 39(10):1386-94, (1986).

8. Anonymous. 1986. Pharmaceutical R\&D Spending by US Industry Hits \$4.1 Billion, Setting Record, as do Sales. P. 5 in Chem. Mark. Rep. February 3, 1986.

9. Saklani A, Kutty SK. Plant-derived compounds in clinical trials. Drug Discov Today. 13(3):161-71,( 2008)

10. Gadgil M, Rao PRS. Nurturing biodiversity: An Indian agenda. Centre for Environment Education, Ahmadabad, India. (1998).

11. Clinical and Laboratory Standards Institute. Performance Standards for Antimicrobial Susceptibility Testing; Twenty-Fourth Informational Supplement. CLSI document M100-S24, 2014. Clinical and Laboratory Standards Institute, 950 West Valley Road, Suite 2500, Wayne, Pennsylvania 19087, USA.

12. Bauer, A.W., Kirby, W.M.M., Serris, J.C. \& Turck, M.. Antibiotic susceptibility testing by a standardized single disc method. Am J Clin Pathol., 45: 493-496, (1966).
13. Kamboj VP Herbal Medicine. Current Science, 78: 35-9, (2000).

14. Ladda PL, Magdum CS. Vitex negundo Linn. Ethnobotany, phytochemistry and pharmacology-A review. International Journal of advances in Pharmacy, Biology and Chemistry.1(1):111-20, (2012).

15. Thati V, Shivannavar CT, Gaddad SM. Vancomycin resistance among methicillin resistant Staphylococcus aureus isolates from intensive care units of tertiary care hospitals in Hyderabad. Indian $J$ Med Res, 134:704-8, (2011).

16. Tarai, B., Das, P. and Kumar, D., Recurrent challenges for clinicians: emergence of methicillin-resistant Staphylococcus aureus, vancomycin resistance, and current treatment options. J Lab Physicians,5(2):71, (2013).

17. Devi PR, Kokilavani R, Poogotha SG. Anti-Microbial activity of the various leaf extracts of Vitex negundo Linn. Ancient science of life. 28(2):15 (2008).

18. Patra JM, Panda SS, Banani Pattanayakand Nabin K Dhal. Validations of Tribal Claims On Vanda Tessellate (Roxb.) Hook. Ex G. Don., Vitex Negundo L. And Holarrhena Antidysenterica Wall.Ex.A.Dc. Through Phytochemical Screening and Antibacterial Activity. Int. J. Pharm. Biol. Sci. 6(8): 593-599, (2015).

19. Kamruzzaman M, Bari SN, Faruque SM. In vitro and in vivo bactericidal activity of Vitex negundo leaf extract against diverse multidrug resistant enteric bacterial pathogens. Asian Pac J Trop Dis, 6(5):352-9 (2013). 